\title{
Symptoms of Primary Hyperparathyroidism in Men and Women: The Same but Different?
}

\author{
Theresia Weber $^{\mathrm{a}}$ Andreas Hillenbrand ${ }^{\mathrm{b}}$ Simon Peth ${ }^{\mathrm{a}}$ Rainer Hummel ${ }^{\mathrm{a}}$ \\ ${ }^{a}$ Department of Endocrine Surgery, Katholisches Klinikum Mainz, Mainz, Germany; \\ ${ }^{\mathrm{b}}$ Department of General and Visceral Surgery, Ulm University, Ulm, Germany
}

\section{Keywords}

Primary hyperparathyroidism · Gender · Symptoms

\begin{abstract}
Introduction and Objective: Typical symptoms for primary hyperparathyroidism (pHPT) include osteoporosis, bone or joint pain, and nephrolithiasis, as well as fatigue, depression, gastritis, and cardiovascular disease. The female:male ratio was 3:1. The aim of this retrospective study was to evaluate the possible influence of gender-specific aspects of these symptoms. Methods: From February 2018 to November 2019, parathyroid surgery was performed in 125 patients with pHPT (age: 23-83 years); 95 (76\%) were female, and 30 (24\%) were male. Preoperatively, a standardized medical history including 7 typical symptoms of pHPT was used for routine clinical documentation according to the StuDoQ Thyroid and Parathyroid Registry. Results: For both groups (mean age: 60.4 years for females and 60.2 years for males), no sex differences were found in serum calcium or parathyroid hormone levels. For females, there was a tendency towards smaller hyperfunctioning parathyroid glands (16.8 vs. $20.5 \mathrm{~mm}$ ) with less weight (1.14 vs. $1.52 \mathrm{~g}$ ) and multiglandular disease. Nephrolithiasis was significantly more frequent in men than in women ( 36.7 vs. $16.8 \%$, respectively; $p=$ $0.036)$, but the difference in hypertension was not significant ( $60 \%$ in men vs. $47.4 \%$ in women, $p=0.230$ ). Women reported significantly more often bone and joint pain $(44.2 \%$ in women vs. $20 \%$ in men, $p=0.053$ ) and depressive episodes (32.6\% in women vs. $10 \%$ in men, $p=0.016)$. Conclusion: $91.2 \%$ of patients transferred for parathyroidectomy de-
\end{abstract}

scribed typical symptoms of pHPT. However, an unexpected high gender difference was found following the analysis of symptoms documented in the StuDoQ Registry.

๑) 2020 S. Karger AG, Basel

\section{Introduction}

Primary hyperparathyroidism (pHPT) is caused by an overproduction of parathyroid hormone (PTH) in relation to serum calcium levels either by a single parathyroid adenoma (85-90\%) or multiple hyperfunctional parathyroid glands (10-15\%), such as double adenomas or parathyroid hyperplasia.

The incidence/prevalence of pHPT amounts to 0.78$1.07 \%$ of the general population in Latin America, the United States, Canada, and Europe. In Canada, about $3.3 \%$ of women and $1.4 \%$ of men are diagnosed with pHPT [1].

The diagnosis of pHPT is proven biochemically by elevated serum calcium levels and correspondingly elevated or high normal PTH levels. Twenty-four-hour urinary calcium measurement is recommended to exclude familial hypocalciuric hypercalcemia. According to the guidelines of the American Association of Endocrine Surgeons (AAES) [2], a careful medical history should assess renal and skeletal manifestations.

From 1990 to 2014, several consensus conferences for the management of asymptomatic pHPT $[3,4]$ recommended parathyroid surgery only for patients with elevated serum calcium levels $(>2.85 \mathrm{mmol} / \mathrm{L})$ or increased 
Table 1. Characteristics of 125 patients undergoing parathyroid surgery for pHPT: medians (ranges) and numbers (\%)

\begin{tabular}{lccc}
\hline & pHPT (total) & Female & Male \\
\hline Patients, $n$ & 125 & 95 & 30 \\
Age, years & $60.4(23-83)$ & $60.4(23-83)$ & $60.2(44-83)$ \\
Serum calcium (normal range 2.1-2.6), mmol/L & $2.75(2.26-3.53)$ & $2.75(2.4-3.53)$ & $2.76(2.26-3.21)$ \\
Parathyroid hormone (normal range 15-78), ng/L & $162.7(47.4-648.2)$ & $163.2(47.4-648.2)$ & $161.1(68.5-412.4)$ \\
Single gland adenoma, $n$ & $115(92.0)$ & $87(91.6)$ & $28(93.3)$ \\
Double adenoma, $n$ & $5(4.0)$ & $4(4.2)$ & $1(3.3)$ \\
Parathyroid hyperplasia, $n$ & $5(4.0)$ & $4(4.2)$ & $1(3.3)$ \\
Additional goiter, $n$ & $34(27.2)$ & $30(31.6)$ & $4(13.3)$ \\
Additional thyroid carcinoma, $n$ & $4(3.2)$ & $4(4.2)$ & $0(0)$ \\
Minimally invasive parathyroidectomy, $n$ & $39(31.2)$ & $29(30.5)$ & $10(33.3)$ \\
Size of the largest parathyroid gland, mm & $17.7(5-45)$ & $16.8(5-40)$ & $20.5(5-45)$ \\
Weight of the largest parathyroid gland, $g$ & $1.23(0.1-8.5)$ & $92(96.1-8.5)$ & $1.52(0.3-8.0)$ \\
Patients cured, $n$ & $122(97.6)$ & & $30(100)$ \\
\hline
\end{tabular}

urinary calcium excretion (>10 mmol/day), osteoporosis, vertebral fractures, presence of nephrolithiasis, or nephrocalcinosis in patients aged $<50$ years. The 4 th International Consensus Conference [3, 4] in 2014 stated that symptoms such as changes in cognition, depression, and reduced quality of life were "nonspecific" and should not be used to recommend parathyroidectomy.

In 2016, the AAES released the first evidence-based guidelines [2] for definitive management of pHPT and strongly recommended parathyroidectomy in patients with neurocognitive and neuropsychiatric symptoms attributable to pHPT.

Both guidelines confirmed that surgery is the only curative treatment for $\mathrm{pHPT}$. High-volume surgeons and hospitals for thyroid and parathyroid surgery had lower complication rates, less reoperations, and better outcomes $[5,6]$.

Until now, gender-specific differences in pHPT were reported only in a limited number of studies [7-9]. The aim of this retrospective study was, therefore, to evaluate typical symptoms of pHPT using a standardized medical history as provided by the StuDoQ Thyroid and Parathyroid Registry of the German Society of General and Visceral Surgery (DGAV) with a special focus on genderspecific differences.

\section{Patients and Methods}

From February 2018 to November 2019, 125 patients with pHPT presented at the Division of Endocrine Surgery, Catholic Hospital, Mainz, Germany. pHPT was diagnosed by hypercalcemia (serum calcium above the normal range of 2.1-2.6 $\mathrm{mmol} / \mathrm{L}$ ) and elevated PTH (normal range $18.4-80.1 \mathrm{pg} / \mathrm{mL}$ ) or PTH levels in the upper normal range with elevated serum calcium.

Basic clinical data were obtained from electronic patients' records. Symptoms at presentation were documented according to the German StuDoQ Thyroid and Parathyroid Registry on the patients' first visit of the outpatient clinic. The StuDoQ Registry de- fined the following items as symptoms of pHPT: fatigue, depression, osteoporosis, bone or joint pain, gastritis, nephrolithiasis or nephrocalcinosis, and hypertension.

\section{Statistical Analysis}

To compare differences between gender outcomes, we used a Mann-Whitney U test. For all analyses, the level of statistical significance was set at $p \leq 0.05$. All statistical analyses were performed on WinSTAT ${ }^{\circledR}$ for Microsoft Excel.

\section{Results}

A total of 125 patients (95 females and 30 males) were included in the present study (Table 1). The mean age at the time of surgery was 60.4 years for females (23-83 years) and 60.2 years for males ( $44-83$ years).

The mean serum calcium levels for female and male patients ( 2.75 and $2.76 \mathrm{mmol} / \mathrm{L}$, respectively) as well as the PTH levels were distributed equally $(163.2 \mathrm{pg} / \mathrm{mL}$ for females and $161.1 \mathrm{pg} / \mathrm{mL}$ for males). Only $24.8 \%$ (31/125) of the patients ( $21.1 \%$ females and $36.7 \%$ males) presented with calcium levels $>0.25 \mathrm{mmol} / \mathrm{L}$ above the upper limit of normal $(2.85 \mathrm{mmol} / \mathrm{L})$, currently recommended by surgical and endocrinological guidelines $[2,3]$.

\section{Parathyroid Surgery}

All of the patients were subjected to parathyroid surgery. In 122 of 125 patients (97.6\%), a parathyroid adenoma (92\%) was successfully removed, and patients were cured. Five patients presented a double adenoma and 5 parathyroid hyperplasia intraoperatively, which were confirmed by histopathology. Three patients had persistent $\mathrm{pHPT}$. In 2 of them, intraoperative and early postoperative ( $24 \mathrm{~h}$ after surgery) PTH declined from 128 to 31.4 $\mathrm{pg} / \mathrm{mL}$ and from 267 to $31 \mathrm{pg} / \mathrm{mL}$ following removal of a parathyroid adenoma. Two weeks postoperatively, PTH was above the upper limit in both patients. In one of these 
Table 2. Symptoms of pHPT in 95 female and 30 male patients

\begin{tabular}{lllrl}
\hline & $\begin{array}{l}\text { pHPT total } \\
(n=125) \\
n(\%)\end{array}$ & $\begin{array}{l}\text { Female } \\
(n=95) \\
n(\%)\end{array}$ & $\begin{array}{l}\text { Male } \\
(n=30) \\
n(\%)\end{array}$ & $p$ value \\
\hline Symptomatic pHPT & $114(91.2)$ & $85(89.5)$ & $29(96.7)$ & \\
Asymptomatic pHPT & $11(8.8)$ & $10(10.5)$ & $1(3.3)$ & 0.559 \\
Fatigue & $60(48.0)$ & $47(49.5)$ & $13(43.3)$ & 0.016 \\
Depression & $34(27.2)$ & $31(32.6)$ & $3(10.0)$ & 0.394 \\
Osteoporosis & $50(40.0)$ & $40(42.1)$ & $10(33.3)$ & $6(20.0)$ \\
Bone or joint pain & $48(38.4)$ & $42(44.2)$ & $11(36.7)$ & 0.033 \\
Nephrolithiasis/nephrocalcinosis & $27(21.6)$ & $16(16.8)$ & $18(60.0)$ & 0.230 \\
Hypertension & $63(50.4)$ & $45(47.4)$ & $3(10.0)$ & 0.433 \\
Gastritis & $18(14.4)$ & $15(15.8)$ & & \\
\hline
\end{tabular}

patients, a small $(5 \mathrm{~mm})$ double adenoma was successfully excised. In the second patient, a 59-year-old female, genetic testing detected a new heterogeneous mutation of the calcium-sensing receptor. A 68-year-old female with persistent $\mathrm{pHPT}$ refused to have any further testing or localization procedures.

In 30 of 95 females (31.6\%) and 4 of 30 males (13.3\%) with pHPT, a lobectomy or total thyroidectomy was performed for additional thyroid nodules. In 4 women, 3 papillary thyroid carcinomas and 1 Hurthle cell carcinoma were found intraoperatively.

Histopathologically, the mean size of the largest hyperfunctioning parathyroid removed was $16.8 \mathrm{~mm}$ (5-40 $\mathrm{mm}$ ) in females and $20.5 \mathrm{~mm}(5-45 \mathrm{~mm})$ in males. Compared to female parathyroid glands with a mean weight of $1.14 \mathrm{~g}(0.1-8.5 \mathrm{~g})$, male parathyroid glands were heavier, with a mean weight of $1.52 \mathrm{~g}(0.3-8.0 \mathrm{~g})$. No postoperative surgical complications such as recurrent nerve palsy or persistent hypoparathyroidism were seen.

\section{Symptoms of pHPT in Female and Male Patients}

Overall, 114 of 125 patients (91.2\%) reported symptoms of pHPT according to the items defined in the StuDoQ Registry (Table 2). Men tended to be more symptomatic (96.7\%) than women (89.5\%).

Biochemically, symptomatic patients showed higher serum calcium levels (average: $2.77 \mathrm{mmol} / \mathrm{L}$ ) than asymptomatic patients $(2.60 \mathrm{mmol} / \mathrm{L})$. PTH levels in symptomatic patients were also higher (mean PTH of $167.2 \mathrm{pg} /$ $\mathrm{mL}$ ) than in asymptomatic patients $(116.8 \mathrm{pg} / \mathrm{mL})$.

Overall, the most commonly reported symptoms of pHPT at initial presentation were hypertension (50.4\%), fatigue (48\%), and osteoporosis (40\%).

Gender differences became evident especially for depression, muscular complaints, nephrolithiasis, and hypertension. Female patients reported 3 times more often depressive symptoms, and antidepressive medication was also prescribed more often than in male patients (32.6 vs. $10.0 \%, p=0.016$ ). Moreover, women complained twice as

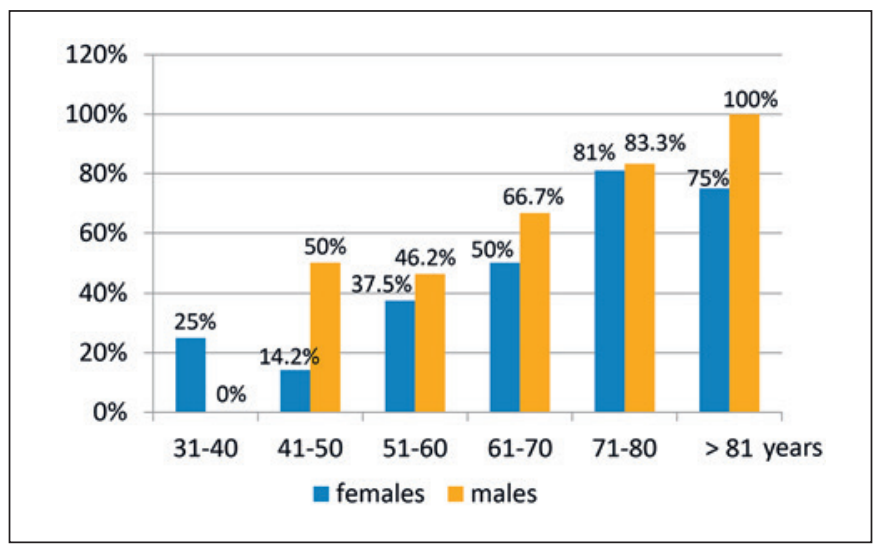

Fig. 1. Hypertension in 95 females and 30 males with pHPT.

much about bone and joint pain than males (44.2 vs. $20.0 \%, p=0.053$ ).

On the other hand, $36.7 \%$ of male patients had a history of kidney stones or nephrocalcinosis, while only $16.8 \%$ of female patients showed a pathologic ultrasound of the kidneys $(p=0.036)$. Of note, abdominal ultrasound was not performed regularly. Hypertension was a frequent finding in females (47.4\%) and even more frequent in males $(60.0 \%)$, but the difference failed to reach statistical significance $(p=0.230)$. Regardless of age (Fig. 1), male patients showed a higher percentage of increased blood pressure when compared to females. At the age of 41-50 years, 3 of 6 men with pHPT (50\%) had hypertension compared to 1 of 7 females (14.2\%). At 51-60 and $61-70$ years of age, hypertension was 8.7 and $16.7 \%$ higher in males, respectively, than in females. Hypertension was only diagnosed when the patients obtained antihypertensive medication.

Figures 2 and 3 compare the percentages of hypertension in our study to the prevalence rates of hypertension in Germany, published by the Robert-Koch-Institute [10]. In women, we found a higher percentage of hypertension, especially in younger females aged $30-44$ years: 


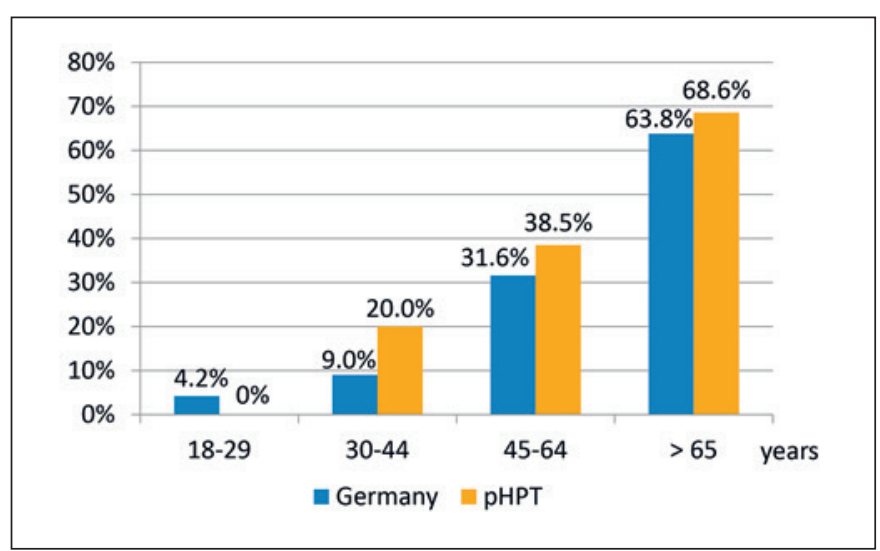

Fig. 2. Prevalence of hypertension in Germany and in 95 females with $\mathrm{pHPT}$

$20 \%$ of our study patients compared to $9 \%$ in a representative sample of the German population. Since we had only 1 male patient in this age group, this finding was not comparable. In the group aged $45-64$ years, $55 \%$ of males with $\mathrm{pHPT}$ were medically treated for hypertension compared to $38.3 \%$ in the German population.

In another 2 male patients, potential symptoms of pHPT were chronic pancreatitis and a rupture of the patellar tendon.

\section{Discussion}

According to the guidelines for the management of asymptomatic pHPT, published in 2014 by Bilezikian et al. [3], only $69.5 \%$ of our cohort patients $(67.4 \%$ female and $76.6 \%$ males) would have been subjected to parathyroid surgery.

In contrast to these findings, the AAES guidelines of 2016 [2] would have recommended parathyroidectomy in $92.8 \%$ of our patients $(91.6 \%$ of females and $96.7 \%$ of males). Interestingly, both guidelines presented a stronger indication to operate on male patients than females. The reason to do so is mainly caused by a quite restrictive definition of symptoms in the consensus guidelines of $2014[3,4]$, which were upgraded by the AAES guidelines for definite management of pHPT [2] with a successful approach to establish an evidence-based guideline. From our point of view, it was not only very important to state that $\mathrm{pHPT}$ remains underdiagnosed and undertreated, but also to refer to neurocognitive and psychiatric symptoms. In the present study, every third female patient was diagnosed with depression or was using antidepressant drugs, which was significantly less noticed in male patients (only 1 of 10 male patients).

In addition, in a German multicenter trial comparing anxiety, depression, and quality of life in pHPT patients

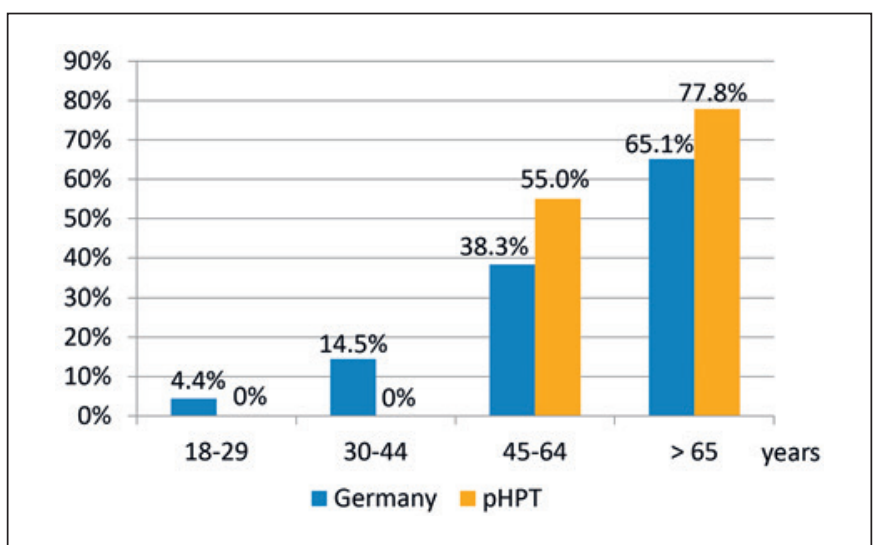

Fig. 3. Prevalence of hypertension in Germany and in 30 males with $\mathrm{pHPT}$.

and a control group with benign thyroid nodules, our group [11] showed that women's preoperative anxiety scores exceeded those of men $(p=0.05)$ and were significantly improved at the 12-month follow-up ( $p<$ 0.001).

Blanchard et al. [12] performed a multicenter study including 116 patients with mild pHPT; using the SF-36 questionnaire, they found that men did not improve their mental component summary. Women with a preoperative mental component summary score $<43.6$ of 100 showed postoperative improvement.

In 1998, Pasieka and Parsons [13] were one of the first endocrine surgeons bringing neuromuscular and psychiatric complaints such as fatigue, weakness, mood swings, and bone and joint pain into the focus of parathyroid research and developed a patient-based surgical outcome tool to examine these symptoms [14].

In a multicenter trial, Pasieka et al. [14] described that the symptom "feeling tired easily" was reported by $45-$ $60 \%$ pHPT patients treated at 3 endocrine centers in Canada, the United States, and Australia [14]. In a study by Roman et al. [15], fatigue was noted in $53 \%$ of 212 patients with pHPT. In our recent series, $48 \%$ of all patients with pHPT reported fatigue, which occurred slightly more often in women $(49.5 \%)$ than men $(43.3 \%)$.

Osteoporosis defined as T scores $\leq 2.5$ at lumbar spine, total hip, or femoral neck by bone densitometry was recognized in $40 \%$ of all patients in our study. It was more prevalent in women (42.1\%) than men (33.3\%). Castellano et al. [8] found osteoporosis much more frequently in postmenopausal women $(59.2 \%)$ than in premenopausal women $(28.1 \%)$ with pHPT.

In a retrospective study, Vodopivec et al. [9] analyzed pre- and postoperative results of bone mineral density in 80 patients (56 women and 24 men) with pHPT undergoing parathyroid surgery. In this study, $25 \%$ of men and $33.9 \%$ of women had osteoporotic preoperative $\mathrm{T}$ scores. 
One year after parathyroidectomy, men had a greater increase in bone mineral density in the femoral neck than women.

In a nested case-control study of the Danish National Data Patient Register [16], published in 2018, on prevalence and risk of vertebral fractures in pHPT patients, 588 patients ( $77 \%$ females and $23 \%$ males) were examined. Vertebral fractures were quite equally distributed in females and males (20.5 and 21.5\%, respectively). After stratification by age, the prevalence differed significantly between sexes $(p<0.01)$. In patients $<50$ and between 50 and 59 years, the prevalence of vertebral fractures in men was almost 3 times, and twice as high in women, respectively. For both sexes, prevalence of vertebral fractures did not correlate with serum calcium or PTH levels.

Even though we did not perform a routine screening for kidney stones or nephrocalcinosis, $36.7 \%$ of our male patients and $16.8 \%$ of the females with pHPT presented with a history of nephrolithiasis or nephrocalcinosis. In 2000, a study on urolithiasis in Germany including a representative sample of 7,500 persons found a prevalence of $4.7 \%$. In males $50-64$ years old, $9.7 \%$ had urinary stones compared to $5.9 \%$ in females [17].

Reid et al. [18] described that younger age and male sex were the only independent predictors for nephrolithiasis in 611 patients with pHPT. A history of nephrolithiasis was present in $10 \%$ of the patients and was associated with higher serum calcium and PTH levels. In this cohort, at presentation, $8.5 \%$ of women and $16.7 \%$ of men had a history of renal stones.

In contrast to our findings which showed no difference in serum calcium levels in patients with or without kidney disease, Pappachan et al. [19] found that a higher peak calcium concentration was an independent predictor for acute kidney injury.

With respect to cardiovascular disease as an indication to perform parathyroid surgery, the current AAES guidelines [2] reported conflicting data for observational studies in mild pHPT. Therefore, they supported only a weak recommendation for offering parathyroidectomy to surgical candidates with mild pHPT and cardiovascular disease. In this study, we found a constantly higher percentage of hypertensive female and male patients with pHPT compared to a representative sample of 7,500 persons of the German population [10].

In 2012, even more striking results were published by Han et al. [7], who performed a retrospective case-control study. They compared patients with pHPT with an average age of 56 years to the general population of New Jersey. In the pHPT group, $48.6 \%$ of females and $57.4 \%$ of males had hypertension. In the New Jersey population, the percentage of hypertension was only $27.2 \%$ for females and $29 \%$ for males. In addition to these findings, prevalence rates of obesity, hyperlipidemia, type 2 diabetes, and coronary artery disease were higher in $\mathrm{pHPT}$ patients than in the general New Jersey population and age-, sex-, and BMI-matched goiter patients. Compared to female patients with pHPT, prevalence rates of obesity, hypertension, and type 2 diabetes were $29.7,15.3$, and $52.6 \%$ higher in male patients, respectively.

In 2018, Pappachan et al. [19] summarized that male gender was a statistically significant predictor for patients receiving parathyroidectomy. Since male patients have larger and heavier adenomas, which can be detected more easily with localization procedures, more often present with kidney stones or nephrocalcinosis, and have higher blood pressure, parathyroid surgery might probably be offered more liberally to them than to women complaining of bone pain, depression, or mood swings, which are more difficult to explore.

The advantage of this study was that the StuDoQ Thyroid and Parathyroid Registry enabled participating hospitals to evaluate symptoms, laboratory tests, and operative strategies of pHPT according to gender and age to relate these results to our studies or current guidelines.

The limitations of our retrospective, single-center study are that kidney ultrasound, bone densitometry, and questionnaires for depression were not performed in all patients. Since patients were already referred to a department of endocrine surgery, the possibility of a selection bias must be addressed, and reported patients were more likely to present with advanced stages of the disease.

\section{Conclusions}

The results of our study suggest that symptoms of pHPT are different in women and men. While women suffer more often from osteoporosis, bone and joint pain, as well as depression, male patients presented higher rates of nephrolithiasis and hypertension. Current guidelines are focusing on both sexes, but maybe a more genderspecific approach to $\mathrm{PHPT}$ seems to be eligible.

\section{Statement of Ethics}

This retrospective study was conducted ethically in accordance with the World Medical Association Declaration of Helsinki. All of the patients provided written informed consent to participate in the StuDoQ Thyroid and Parathyroid DGAV Registry, and agreed to data evaluation and publication. Ethics committee approval was not required since the study involved only retrospective data of our own patient cohort. 


\section{Disclosure Statement}

T.W. received speakers' honoraria from Sanofi-Aventis, Germany, and Novo Nordisk, Germany, as well as expert testimonials from Sanofi-Aventis, Germany, and Shire Pharmaceutical, Germany. R.H. received a travel grant from Medtronic, Germany. A.H. and S.P. have no conflicts of interest to declare.

\section{Author Contributions}

T.W., S.P., and R.H.: data collection and analysis. A.H.: statistical analysis. T.W.: drafting of the manuscript. All of the authors approved the manuscript.

\section{References}

1 Khan AA, Hanley DA, Rizzoli R, Bollerslev J, Young JE, Rejnmark L, et al. Primary hyperparathyroidism: Review and recommendations on evaluation, diagnosis, and management. A Canadian and international consensus. Osteoporos Int. 2017 Jan;28(1):1-19.

2 Wilhelm SM, Wang TS, Ruan DT, Lee JA, Asa SL, Duh QY, et al. The American Association of Endocrine Surgeons Guidelines for definitive management of primary hyperparathyroidism. JAMA Surg. 2016 Oct;151(10):95968.

3 Bilezikian JP, Brandi ML, Eastell R, Silverberg SJ, Udelsman R, Marcocci C, et al. Guidelines for the management of asymptomatic primary hyperparathyroidism: Summary statement from the Fourth International Workshop. J Clin Endocrinol Metab. 2014 Oct;99(10): 3561-9.

4 Silverberg SJ, Clarke BL, Peacock M, Bandeira F, Boutroy S, Cusano NE, et al. Current issues in the presentation of asymptomatic primary hyperparathyroidism: Proceedings of the Fourth International Workshop. J Clin Endocrinol Metab. 2014 Oct;99(10):3580-94.

5 Abdulla AG, Ituarte PH, Harari A, Wu JX, Yeh MW. Trends in the frequency and quality of parathyroid surgery: Analysis of 17,082 cases over 10 years. Ann Surg. 2015 Apr; 261(4):746-50.

6 Mitchell J, Milas M, Barbosa G, Sutton J, Berber E, Siperstein A. Avoidable reoperations for thyroid and parathyroid surgery: effect of hospital volume. Surgery. 2008 Dec;144(6): 899-906.
7 Han D, Trooskin S, Wang X. Prevalence of cardiovascular risk factors in male and female patients with primary hyperparathyroidism. J Endocrinol Invest. 2012 Jun;35(6):548-52.

8 Castellano E, Attanasio R, Boriano A, Pellegrino M, Garino F, Gianotti L, et al. Sex difference in the clinical presentation of primary hyperparathyroidism: influence of menopausal status. J Clin Endocrinol Metab. 2017 Nov;102(11):4148-52.

9 Vodopivec DM, Silva AM, Garcia-Banigan DC, Christakis I, Stewart A, Schwarz K, et al. Gender differences in bone mineral density in patients with sporadic primary hyperparathyroidism. Endocrinol Diabetes Metab. 2018 Sep;1(4):e00037.

10 Neuhauser H, Kuhnert R, Born S. 12-MonatsPrävalenz von Bluthochdruck in Deutschland. J Health Monitor. 2017;2(1):57-63.

11 Weber T, Eberle J, Messelhäuser U, Schiffmann L, Nies C, Schabram J, et al. Parathyroidectomy, elevated depression scores, and suicidal ideation in patients with primary hyperparathyroidism: Results of a prospective multicenter study. JAMA Surg. 2013 Feb; 148(2):109-15.

12 Blanchard C, Mathonnet M, Sebag F, Caillard C, Kubis C, Drui D, et al. Quality of life is modestly improved in older patients with mild primary hyperparathyroidism postoperatively: Results of a prospective multicenter study. Ann Surg Oncol. 2014 Oct;21(11): 3534-40.

13 Pasieka JL, Parsons LL. Prospective surgical outcome study of relief of symptoms following surgery in patients with primary hyperparathyroidism. World J Surg. 1998 Jun; 22(6):513-8.
14 Pasieka JL, Parsons LL, Demeure MJ, Wilson S, Malycha P, Jones J, et al. Patient-based surgical outcome tool demonstrating alleviation of symptoms following parathyroidectomy in patients with primary hyperparathyroidism. World J Surg. 2002 Aug;26(8):942-9.

15 Roman SA, Sosa JA, Pietrzak RH, Snyder PJ, Thomas DC, Udelsman R, et al. The effects of serum calcium and parathyroid hormone changes on psychological and cognitive function in patients undergoing parathyroidectomy for primary hyperparathyroidism. Ann Surg. 2011 Jan;253(1):131-7.

16 Ejlsmark-Svensson H, Bislev LS, Lajlev S, Harsløf T, Rolighed L, Sikjaer T, et al. Prevalence and risk of vertebral fractures in primary hyperparathyroidism: A nested case-control study. J Bone Miner Res. 2018 Sep;33(9): 1657-64.

17 Hesse A, Brändle E, Wilbert D, Köhrmann KU, Alken P. Study on the prevalence and incidence of urolithiasis in Germany comparing the years 1979 vs. 2000. Eur Urol. 2003 Dec; 44(6):709-13.

18 Reid L, Muthukrishnan B, Patel D, Crane M, Akyol M, Thomson A, et al. Presentation, diagnostic assessment and surgical outcomes in primary hyperparathyroidism: A single centre's experience. Endocr Connect. 2018 Aug; 7(10):1105-15.

19 Pappachan JM, Elnaggar MN, Sodi R, Jbeili K, Smith PR, Lahart IM. Primary hyperparathyroidism: Findings from the retrospective evaluation of cases over a 6-year period from a regional UK centre. Endocrine. 2018 Oct; 62(1):174-81. 